\title{
Long-Term Survival after High-Dose Chemotherapy Followed by Peripheral Stem Cell Rescue for High-Risk, Locally Advanced/ Inflammatory, and Metastatic Breast Cancer
}

\author{
A. VanderWalde ${ }^{1}$, W. Ye ${ }^{2}$, P. Frankel ${ }^{2}$, D. Asuncion ${ }^{5}$, L. Leong ${ }^{1}$, T. Luu ${ }^{1}$, R. Morgan ${ }^{1}$, P. \\ Twardowski $^{1}$, M. Koczywas ${ }^{1}$, R. Pezner ${ }^{3}$, I. B. Paz ${ }^{4}$, K. Margolin ${ }^{6}$, J. Wong ${ }^{3}$, J. H. \\ Doroshow $^{7}$, S. Forman ${ }^{8}$, S. Shibata ${ }^{1}$, and G. Somlo ${ }^{1}$
}

${ }^{1}$ Department of Medical Oncology and Therapeutics Research, City of Hope Comprehensive Cancer Center, Duarte, California ${ }^{2}$ Department of Information Sciences, City of Hope Comprehensive Cancer Center, Duarte, California ${ }^{3}$ Department of Radiation Oncology, City of Hope Comprehensive Cancer Center, Duarte, California ${ }^{4}$ Department of General Oncologic Surgery, City of Hope Comprehensive Cancer Center, Duarte, California ${ }^{5}$ Medical Oncology and Hematology, Kaiser Permanente, Irvine, California ${ }^{6}$ Seattle Cancer Care Alliance, Seattle, Washington ${ }^{7}$ Division of Cancer Treatment, National Cancer Center, Bethesda, Maryland ${ }^{8}$ Department of Hematology and Hematopoietic Cell Transplantation

\begin{abstract}
Patients with high-risk locally advanced/inflammatory and oligometastatic ( $₫$ sites) breast cancer frequently relapse or experience early progression. High-dose chemotherapy combined with peripheral stem cell rescue may prolong progression-free survival/relapse-free survival (PFS/RFS) and overall survival (OS). In this study, patients initiated high-dose chemotherapy with STAMP-V (carboplatin, thiotepa, and cyclophosphamide), ACT (doxorubicin, paclitaxel, and cyclophosphamide), or tandem melphalan and STAMP-V. Eighty-six patients were diagnosed with locally advanced/inflammatory ( 17 inflammatory) breast cancer, and 12 were diagnosed with oligometastatic breast cancer. Median follow-up was 84 months (range, 6-136 months) for patients with locally advanced cancer and 40 months (range, 24-62 months) for those with metastatic cancer. In the patients with locally advanced cancer, 5-year RFS and OS were 53\% (95\% CI, 41\%-63\%) and 71\% (95\% CI, 60\%-80\%), respectively, hormone receptors were positive in $74 \%$, and HER 2 overexpression was seen in $23 \%$. In multivariate analysis, hormone receptor-positive disease and lower stage were associated with better 5-year RFS (60\% for ER [estrogen receptor]/PR [progesterone receptor]-positive versus 30\% for ER/PR-negative; $P<.01$ ) and OS (83\% for ER/PR-positive versus $38 \%$ for ER/PR-negative; $P<.001)$. In the patients with metastatic cancer, 3-year PFS and OS were 49\% (95\% CI, 19\%-73\%) and 73\% (95\% CI, 38\%91\%), respectively. The favorable long-term RFS/PFS and OS for high-dose chemotherapy with peripheral stem cell rescue in this selected patient population reflect the relative safety of the procedure and warrant validation in defined subgroups through prospective, randomized, multiinstitutional trials.
\end{abstract}

\footnotetext{
(C) 2012 American Society for Blood and Marrow Transplantation. Published by Elsevier Inc. All rights reserved. Correspondence and reprint requests: George Somlo, City of Hope National Medical Center, 1500 East Duarte Road, Duarte, CA 91010 (gsomlo@coh.org).

Data from this study were presented at the annual meeting of the American Society of Clinical Oncology, Chicago, Illinois, June 3-7, 2011.

Financial disclosure: All other authors report no conflicts of interest.
} 


\section{Keywords}

High-dose chemotherapy; Autologous peripheral blood transplantation; Adjuvant therapy; Highrisk breast cancer

\section{INTRODUCTION}

Breast cancer is the leading cause of cancer death in women worldwide [1]. In patients with locally advanced breast cancer (LABC), the risk of recurrence is high, and the estimated 10year overall survival (OS) of patients with stage III disease is only 50\% [2]. In patients with $\geq 10$ axillary nodal metastases (stage IIIC), the risk of relapse is even higher; 15-year disease-free survival (DFS) is $26 \%$ with adjuvant anthracycline-based chemotherapy [3]. Patients with inflammatory breast cancer (IBC) have the worst prognosis, with a 5-year OS of $40 \%$ [4]. Once distant metastases develop, the median survival is $18-24$ months, with only $14.5 \%$ of patients surviving for 5 years $[4,5]$.

High-dose chemotherapy (HDCT) followed by autologous peripheral blood progenitor cell (PBPC) rescue has been compared with standard-dose chemotherapy in trials, but the approach remains controversial. Data from trials of some of the earliest regimens suggested an unacceptably high early mortality rate, up to 10\% [6]. Although reports of 3-year RFS in high-risk breast cancer approached $70 \%$ in early-phase trials and retrospective registry trials [6-12], possible selection bias in the determination of who receives HDCT has been demonstrated [13]. Randomized controlled trials (RCTs) have demonstrated a significantly improved event-free survival (EFS) at 3, 4, and 5 years after treatment with HDCT; however, no OS benefit has been apparent [14].

We have conducted a number of HDCT protocols with PBPC rescue aimed at either LABC/ IBC patients (in accordance with American Joint Committee on Cancer version 7, with an estimated risk of progression of $>50 \%$ at 5 years), or focusing on oligometastatic stage IV breast cancer (MBC) to determine the effect on relapse-free survival/progression-free survival (RFS/PFS) and OS in patients with high-risk or oligometastatic MBC, as well as to delineate safety. Here we report long-term safety and efficacy data for patients treated prospectively with HDCT regimens either on a randomized trial comparing doxorubicin, paclitaxel, and cyclophosphamide (ACT) $[15,16]$ versus carboplatin, thiotepa, and cyclophosphamide (STAMP-V) [17] or on a subsequent trial of tandem melphalan and STAMP-V [18].

\section{METHODS}

\section{Patients}

Patients treated with HDCT followed by PBPC rescue on 2 sequentially developed protocols at City of Hope Medical Center between 1999 and 2009 are included in this report. The first HDCT protocol randomized patients with LABC/IBC (stage IIIA-C) between a novel regimen of ACT and STAMP-V, with enrollment starting in February 1999. The ACT arm was closed in December 2003 due to toxicity concerns (primarily mucositis requiring narcotics). All study patients subsequently received STAMP-V through December 2007. The study included a second randomization between bisphosphonate pamidronate versus no pamidronate. The second protocol, which consisted of high-dose melphalan followed by STAMP-V as tandem HDCT, enrolled patients with LABC/IBC and MBC (with at least partial response) between September 2005 and November 2009. All patients participating in these City of Hope Medical Center Institutional Review Board-approved trials provided written voluntary informed consent. 
On both protocols, conventional adjuvant chemotherapy (maximum allowed dose of doxorubicin, $\_40 \mathrm{mg} / \mathrm{m}^{2}$ ) was required $<12$ months before enrollment for LABC/IBC. Stage IV patients were limited to those who had oligometastatic disease ( $\_$organ sites involved with metastases, regardless of the number of lesions per organ) before induction therapy and $₫$ total residual lesions after induction chemotherapy (eg, 3 bone lesions: 1 bone, 1 liver, and 1 skin lesion) within 6 months before enrollment. Inclusion criteria for both studies included a Karnofsky performance status of $\geq 80 \%$, age $\$ 65$ years, adequate cardiac (left ventricular ejection fraction $255 \%$ ), renal (creatinine clearance $\geq 70 \mathrm{~mL} / \mathrm{min}$ ), hepatic (serum aspartate aminotransferase and alanine aminotransferase $\_$times the upper limit of normal), and pulmonary function, as well as adequate blood counts (neutrophyl count of $1000 \mu / \mathrm{L}$ and platelet count of $100,000 \mu / \mathrm{L}$ ).

\section{Treatment}

Induction chemotherapy and PBPCs-All patients with LABC/IBC received definitive treatment before enrollment, including surgery and chemotherapy. Patients with stage IV disease completed therapy for their metastatic breast cancer $\geq 4$ weeks before enrollment. Methods of PBPC collection have been described previously [11]. The minimum cell dose required for transplantation was $2 \times 10^{6} \mathrm{CD} 34^{+}$cells $/ \mathrm{kg}$ with the single HDCT regimens and $4 \times 10^{6} \mathrm{CD} 34^{+}$cells $/ \mathrm{kg}$ with the tandem regimen.

HDCT regimens-The ACT regimen consisted of doxorubicin $41.25 \mathrm{mg} / \mathrm{m}^{2}$ daily given as a continuous i.v. infusion over 96 hours on day -9 to day -6 , cyclophosphamide $100 \mathrm{mg} /$ $\mathrm{kg}$ i.v. on day -5 , and paclitaxel $725 \mathrm{mg} / \mathrm{m}^{2}$ i.v. over 24 hours on day -4 . On day $-2,25 \%$ of PBPCs were reinfused, in view of our previous published experience suggesting that potentially "sacrificial" early reinfusion of a fraction of PBPCs leads to earlier granulocyte recovery [19]. The remaining 75\% of PBPCs were reinfused on day 0 . Granulocyte colony stimulating factor (G-CSF) at $5 \mu \mathrm{g} / \mathrm{kg}$ i.v. was started on day 0 .

The other 2 regimens involved STAMP-V alone (in the first protocol) or after melphalan as part of a tandem transplant (second protocol). STAMP-V comprised cyclophosphamide 1.5 $\mathrm{g} / \mathrm{m}^{2} /$ day, carboplatin $200 \mathrm{mg} / \mathrm{m}^{2} /$ day, and thiotepa $125 \mathrm{mg} / \mathrm{m}^{2} /$ day given as a continuous i.v. infusion for 96 hours on days -7 through -4 . For the single transplant regimen, $25 \%$ of PBPCs were reinfused on day -2 , and the remaining $75 \%$ were infused on day 0 . G-CSF 5 $\mu \mathrm{g} / \mathrm{kg}$ i.v. was started on day 0 .

The tandem protocol allowed for an infusion of trastuzumab $8 \mathrm{mg} / \mathrm{kg}$, for patients with HER2-positive tumors, administered on day -2 of cycle 1 . All patients received melphalan $150 \mathrm{mg} / \mathrm{m}^{2}$ i.v. over 30 minutes on day -1 . On day $0,50 \%$ of PBPCs were reinfused, and daily G-CSF administration was begun. After recovery of marrow function, patients proceeded with cycle 2 , consisting of STAMP-V. Trastuzumab was given on day -7 if the patient's tumor was HER2-positive. PBPCs were reinfused on day -2 (12.5\% of total) and day $0(37.5 \%)$, with administration of G-CSF, as before, starting on day 0 .

Additional therapies-In patients with LABC/IBC, those with estrogen receptor (ER)and/or progesterone receptor (PR)-positive tumors were started on tamoxifen $20 \mathrm{mg}$ within 4-6 weeks of the day of HDCT (day 0), which continued until recurrence or the completion of 5 years of therapy. Patients randomized to the pamidronate arm received the drug at a dose of $90 \mathrm{mg}$ i.v. every 4 weeks for 24 months, with the first dose given within 4-6 weeks of day 0 . Local-regional radiation therapy, including the primary site and supraclavicular and axillary nodal areas within 6-8 weeks of day 0 of HDCT, was recommended for all patients. 
For patients receiving the tandem regimen, trastuzumab and antiestrogen therapy (as appropriate) were prescribed. Patients with $\mathrm{LABC} / \mathrm{IBC}$ were to undergo local-regional radiation therapy, initiated 6-8 weeks from cycle 2 . Treatment of internal mammary nodes was at the discretion of the treating radiation oncologist, as was the use of intensitymodulated radiation therapy.

Patients with stage IV disease received consolidative radiotherapy within 6-8 weeks of cycle 2 with standard intensity-modulated or helical tomotherapy to all visible, responding metastatic lesions present before HDCT. Sites demonstrating complete response were not treated. All visible sites received 180-220 cGy/day, to a total dose of 4000-5000 cGy.

\section{Statistical Considerations}

Survival outcomes included RFS/PFS and OS, calculated from time of initial PBPC rescue. For RFS/PFS, the events included death or disease recurrence (disease progression for patients with stage IV disease), whichever came first. Data for patients who did not experience disease recurrence or progression and were still alive were censored at the date of last follow-up. For OS, data for patients who were still alive were censored at the date of last follow-up. The log-rank test was used in univariate survival analyses to test the associations between survival outcomes and various clinical variables. Survival rates were estimated using the Kaplan-Meier method. Cox regression analysis was used to calculate hazard ratios (HRs) and their 95\% confidence intervals (CIs) in univariate and multivariate analyses.

\section{RESULTS}

\section{Patient Characteristics}

A total of 98 patients ( 71 on the single trial and 27 on the tandem trial) were evaluable for toxicity and outcome. Follow-up data were collected up to the end of 2010. Patient characteristics are presented in Table 1.

On the single regimen, 19 patients received ACT before this arm was closed because of toxicities, and 52 patients received HDCT with STAMP-V. On the tandem regimen, 27 patients received melphalan, but 6 of these patients (22\%) were unable to receive the second cycle with STAMP-V because of either death (1 patient, due to sepsis) or disease progression (5 patients). On the tandem regimen, patients with HER2-positive breast cancer were also treated with trastuzumab. All patients received some form of radiation to affected areas as part of treatment.

\section{Efficacy}

The median follow-up time was 84 months (range, 6-136 months) for living patients with LABC/IBC and 40 months (range, 24-62 months) for those with MBC. In patients with LABC, 5-year RFS and OS were 53\% (95\%CI 41\%-63\%) and 71\% (95\% CI 60\%-80\%), respectively. Separating out patients by LABC stage using American Joint Committee on Cancer version 7 criteria, the 5-year RFS was $84 \%$ for stage IIIA, $63 \%$ for stage IIIB, and $45 \%$ for stage IIIC, and corresponding 5-year OS was 92\%, 89\%, and 65\% (Figure 1). In patients with stage IV oligometastatic disease, 3-year PFS and OS were 49\% (95\% CI, $19 \%-73 \%$ ) and $73 \%$ (95\% CI, 38\%-91\%), respectively (Figure 2).

Disease and treatment characteristics were examined for association with RFS and OS in patients with stage III cancer (Table 2). Those with stage IIIC disease had a significantly worse RFS (HR, 4.76; $P=.017$ ) and marginally significantly worse OS (HR, 3.42; $P=.073$ ) than those with stage IIIA disease. ER/PR-positive disease was significantly associated with 
better 5-year RFS $(60 \%$ versus $30 \% ; P<.01)$ and OS $(83 \%$ versus $38 \% ; P<.001)$ than ER/ PR-negative disease. No other factor, including HER2 status, tumor grade, histopathology, neoadjuvant chemotherapy, and type of HDCT, demonstrated statistically significant differences in either RFS or OS. In multivariate Cox regression models, disease stage (IIIC versus IIIA/B) and ER/PR status continued to be significantly associated with RFS and OS (Table 3).

\section{Toxicity}

Table 4 shows a breakdown of grade $\geq 3$ toxicities possibly related to treatment, stratified by HDCT regimen. There were no unexpected hematologic toxicities or primary or secondary graft failures, and none of the patients developed myelodysplasia or secondary hematologic malignancies.

Both protocols were associated with a $\geq 90 \%$ rate of grade $\geq 3$ cytopenia, as would be expected in am HDCT-treated patient population. Similarly, high rates of electrolyte abnormalities ( $84 \%$ for ACT, $50 \%$ for STAMP-V, and $44 \%$ for melphalan + STAMP-V) and febrile neutropenia $(84 \%, 48 \%$, and $52 \%$, respectively) were noted. ACT was additionally associated with severe mucositis (84\%) and esophagitis (79\%). STAMP-V was better tolerated overall, with the most common other grade $\geq 3$ toxicities, including nausea/ vomiting (21\%) and infection (16\%). Specifically, in the group receiving melphalan + STAMP-V, common high-grade toxicities included anorexia/weight loss $(41 \%)$, nausea/ vomiting (37\%), mucositis (33\%), infection/sepsis (30\%), and diarrhea (19\%). One patient died of melphalan HDCT-related sepsis on the tandem protocol, and 1 patient developed papillary thyroid carcinoma after tandem HDCT.

\section{DISCUSSION}

We have reported the outcomes and toxicities of HDCT regimens followed by stem cell rescue that were conducted under 2 sequentially developed protocols in patients with highrisk breast cancer (treated with ACT versus STAMP-V HDCT on the first trial and melphalan + STAMP-V on the second trial) and who had oligometastatic disease (treated with melphalan + STAMP-V tandem regimens). Our data compare favorably with data in historical controls using standard-dose chemotherapy for the treatment of high-risk cancer. Our median follow-up time is longer than that in most previous large randomized trials of HDCT in high-risk populations. According to data from the National Cancer Data Base for patients diagnosed in 2001-2002, the 5-year OS rate for patients with stage IIIC breast cancer from time of diagnosis is only 49\% [4]. This contrasts with our cohort, which displayed a 5-year survival rate of $65 \%$ in such cancers. This confirms our previous observations [12], and the results are encouraging given the high rate of IBC seen in our cohort (17 IBC/86 LABC), given that most other previous HDCT trials excluded patients with IBC. The favorable safety data with our approach clearly contributed to the overall positive outcome, although we must be cautious when interpreting data generated from a single institutional, nonrandomized, retrospective dataset. The relative safety of our approach similarly contributed to our 3-year OS rate of 73\% with tandem HDCT in patients with oligometastatic disease, which compares favorably with the $30.1 \% 3$-year survival rate seen in all patients with stage IV breast cancer in the general population $[4,18,20]$. We found no differences in outcome based on disease site (visceral versus nonvisceral), possibly due to the limited number of cases in our series. The relatively small number of selected metastatic breast cancer patients in our series, as well as the lack of matched controls, does not allow for generalized conclusions. Also possibly due to our small sample size, we found no statistically significant benefits of the use of adjuvant bisphosphonates, or for the use of tandem versus single regimens (data not shown). Further testing of both concepts remains worthwhile. Given the time frame of the accrual of most of our patients with LABC/IBC on 
the first trial, trastuzumab was prescribed to only 5 of the 20 HER2-positive patients (data not shown); thus, we can postulate that PFS and OS might have been improved with greater use of trastuzumab.

Previous studies of HDCT have been plagued by concerns of selection bias and a lack of RCTs. However, in the past decade, long-term follow-up data have been released from several RCTs in both LABC and metastatic disease [21]. In high-risk primary disease, 15 trials involving HDCT have been reported [22-36]. The study finding the greatest benefit from HDCT was the West German Study Group (WSG) AM-01 Study [27]. Of 403 patients with at least 9 positive nodes, 201 received tandem cycles of high-dose epirubicincyclophosphamide-thiotepa with PBPC rescue after each cycle, and 202 received conventional treatment with epirubicin-cyclophophamide and CMF. After a median followup of 48.6 months, 4-year EFS was $60 \%$ in the high-dose group and $44 \%$ in the conventional group $(P<.001)$. Alone among randomized trials of HDCT, the WSG AM-01 Study also found a statistically significant OS advantage (4-year OS, $75 \%$ versus $70 \% ; P=.02$ ) [27].

RFS/EFS benefits have been reported in 2 other HDCT trials, 1 by Rodenhuis et al. [29] using cyclophosphamide, thiotepa, and carboplatin, and the other by Roche et al. [36] using cyclophosphamide, mitoxantrone, and melphalan. Neither trial demonstrated an OS benefit after a median follow-up of 7 years and 2.75 years, respectively (although the Dutch trial reported an OS advantage for the subset of triple-negative patients [29]). These trials had differing inclusion criteria, with 1 trial requiring 4 or more positive nodes, and the other requiring more than 7 positive nodes. Whereas additional RTCs have shown trends in favor of HDCT $[22,31,33,34]$ compared with conventional-dose chemotherapy in mature followup, other trials reported less-favorable results [6,23-26,30-32,35].

A Cochrane Reviews meta-analysis of 5111 patients in 13 RCTs performed in 2006 found that a significant EFS benefit was seen in favor of the HDCT arms at various follow-up points, though without OS differences. However, treatment-related mortality was higher in the HDCT arms than in the conventional dosing arms (2.5\% versus $0.1 \%)$ [37]. A more recent meta-analysis of 15 trials by Berry et al. [38] included data on a total of 6210 patients. An absolute EFS benefit of $13 \%$ was seen in favor of HDCT (HR, $0.87 ; P=.001)$ after a median follow-up of 6 years, with a nonsignificant trend toward an OS benefit (HR, 0.94; $P$ $=.13$ ). Because of the heterogeneity of dose-intensity regimens used in these trials, the authors also measured outcome using summation dose-intensity methodology. After adjusting for covariates, higher dose intensity was associated with decreased rate of recurrence (HR, 0.85 per 1 unit increase; $P<.001$ ), as well as with a nonsignificantly increased OS (HR, $0.91 ; P=.033)$.

Various investigators also have performed subgroup analyses to determine whether any factors might lead to higher efficacy of HDCT in high-risk disease. Gluz et al. [39], in a retrospective analysis of the WSG AM-01 Study, found that the benefits of HDCT appeared most pronounced in patients with a basal-like phenotype (ER/PR-negative, HER2-negative, and basal cytokeratin-positive) and in those with grade 3 tumors. Others have speculated that patients with triple-negative disease are the most likely to receive a benefit from HDCT [40]. Although the patients in our cohort with ER/PR-positive tumors did significantly better than those with ER/PR-negative tumors, some of the relatively long DFS seen in the former group is likely due to the fact that patients with ER/PR-positive cancer tend to do better overall than patients with triple-negative disease. Patients with lower-stage (IIIA and B) disease (driven primarily by the number of lymph nodes, except in IBC) did demonstrate better RFS/OS. Interestingly, patients with high-grade tumors did not do worse than those with lower-grade tumors in this study. This might have been due to small sample size, or perhaps high-grade disease was more responsive to HDCT. Berry et al. [38] did not find any 
subset of patients who clearly benefited more than others from HDCT, although molecular markers were largely unexamined in that study.

In metastatic breast cancer, in addition to selective phase II trials [18], a number of RCTs also have been performed in the last decade [41-47]. Generally, these studies found improved PFS/EFS in favor of HDCT, with a median additional EFS of 1-8 months. In addition, 1 of these trials demonstrated an OS benefit of $>2$ years (median OS for HDCT versus standard, 44 months versus 19 months; $P=.02$ ) [43], although the other RCTs failed to show an OS benefit. A Cochrane Database meta-analysis performed in 2004 including 6 trials and 850 patients found a significant EFS benefit at both 1 and 5 years, with a trend toward an OS benefit starting at 5 years $(P=.08)$. However, treatment-related mortality was higher in the HDCT arm than in the conventional treatment arm (3.5\% versus 0.4\%) [14]. Treatment-related mortality was zero in the small subset of patients with MBC in our cohort. In a recent meta-analysis of 6 RCTs for MBC, Berry et al. [48] reported on the outcome of 866 patients. As in high-risk disease, the authors found a statistically significant improvement in PFS ( 0.91 year versus 0.69 year; $P<.001)$, but not in OS $(2.16$ years versus 2.02 years; $P=.08$ ). The authors were unable to identify any subgroup of patients that showed a clear benefit from HDCT. Again, molecular markers were largely unexamined in that study.

\section{CONCLUSION}

HDCT combined with PBPC rescue was safe in our long-term experience and generally effective in decreasing the risk of disease recurrence or disease progression in patients with high-risk or oligometastatic breast cancer. Not all HDCT regimens are created equal, however; the ACT regimen was poorly tolerated and holds little promise in future HDCT trials. The favorable long-term PFS/RFS and OS in our highly selected patient population warrant further assessment. Validation should be pursued in well-defined subgroups of patients, both molecularly defined and biologically selected. The former might include, for example, those with triple-negative phenotype and/or basal/claudin-low genotype, whereas the latter might include premenopausal patients, patients with inflammatory breast cancer, those with residual disease after neoadjuvant chemotherapy, and those with responding oligometastatic nonvisceral metastatic disease. These approaches will be best tested in prospective, randomized, multi-institutional trials.

\section{Acknowledgments}

We thank Carol Wuenschell, PhD, for editorial assistance with the manuscript.

This work was supported by grants from Amgen and Bristol-Myers Squibb, and by grants from the National Institutes of Health (M01 RR00043 and CA 33572). George Somlo received research support for this project from Amgen and Bristol-Myers Squibb. A. VanderWalde had no relationships to disclose throughout the research and preparation of the manuscript, but has since become employed by Amgen.

\section{REFERENCES}

1. Jemal A, Bray F, Center MM, et al. Global cancer statistics. CA Cancer J Clin. 2011; 61:69-90. [PubMed: 21296855]

2. Woodward WA, Strom EA, Tucker SL, et al. Changes in the 2003 American Joint Committee on Cancer staging for breast cancer dramatically affect stage-specific survival. J Clin Oncol. 2003; 21:3244-3248. [PubMed: 12947058]

3. Montero AJ, Rouzier R, Lluch A, et al. The natural history of breast carcinoma in patients with > or $=10$ metastatic axillary lymph nodes before and after the advent of adjuvant therapy: a multiinstitutional retrospective study. Cancer. 2005; 104:229-235. [PubMed: 15937910] 
4. Edge, S.; Byrd, D.; Compton, C., et al., editors. AJCC Cancer Staging Manual. 7th ed.. New York: Springer-Verlag; 2010. American Joint Committee on Cancer; p. 438

5. Robertson FM, Bondy M, Yang W, et al. Inflammatory breast cancer: the disease, the biology, the treatment. CA Cancer J Clin. 2010; 60:351-375. [PubMed: 20959401]

6. Peters WP, Ross M, Vredenburgh JJ, et al. High-dose chemotherapy and autologous bone marrow support as consolidation after standard-dose adjuvant therapy for high-risk primary breast cancer. $\mathbf{J}$ Clin Oncol. 1993; 11:1132-1143. [PubMed: 8501500]

7. Antman KH, Rowlings PA, Vaughan WP, et al. High-dose chemotherapy with autologous hematopoietic stem-cell support for breast cancer in North America. J Clin Oncol. 1997; 15:18701879. [PubMed: 9164197]

8. Gianni AM, Siena S, Bregni M, et al. Efficacy, toxicity, and applicability of high-dose sequential chemotherapy as adjuvant treatment in operable breast cancer with 10 or more involved axillary nodes: five-year results. J Clin Oncol. 1997; 15:2312-2321. [PubMed: 9196145]

9. Somlo G, Doroshow JH, Forman SJ, et al. High-dose chemotherapy and stem-cell rescue in the treatment of high-risk breast cancer: prognostic indicators of progression-free and overall survival. $\mathrm{J}$ Clin Oncol. 1997; 15:2882-2893. [PubMed: 9256132]

10. Tomas JF, Perez-Carrion R, Escudero A, et al. Results of a pilot study of 40 patients using highdose therapy with hematopoietic rescue after standard-dose adjuvant therapy for high-risk breast cancer. Bone Marrow Transplant. 1997; 19:331-336. [PubMed: 9051242]

11. Somlo G, Simpson JF, Frankel P, et al. Predictors of long-term outcome following high-dose chemotherapy in high-risk primary breast cancer. Br J Cancer. 2002; 87:281-288. [PubMed: 12177795]

12. Somlo G, Frankel P, Chow W, et al. Prognostic indicators and survival in patients with stage IIIB inflammatory breast carcinoma after dose-intense chemotherapy. J Clin Oncol. 2004; 22:1839_ 1848. [PubMed: 15143076]

13. Garcia-Carbonero R, Hidalgo M, Paz-Ares L, et al. Patient selection in high-dose chemotherapy trials: relevance in high-risk breast cancer. J Clin Oncol. 1997; 15:3178-3184. [PubMed: 9336353]

14. Farquhar C, Marjoribanks J, Basser R, et al. High-dose chemotherapy and autologous bone marrow or stem cell transplantation versus conventional chemotherapy for women with early poor prognosis breast cancer. Cochrane Database Syst Rev. 2005; 20 CD003139.

15. Henderson IC, Berry DA, Demetri GD, et al. Improved outcomes from adding sequential paclitaxel but not from escalating doxorubicin dose in an adjuvant chemotherapy regimen for patients with node-positive primary breast cancer. J Clin Oncol. 2003; 21:976-983. [PubMed: 12637460]

16. Somlo G, Doroshow JH, Synold T, et al. High-dose paclitaxel in combination with doxorubicin, cyclophosphamide and peripheral blood progenitor cell rescue in patients with high-risk primary and responding metastatic breast carcinoma: toxicity profile, relationship to paclitaxel pharmacokinetics and short-term outcome. Br J Cancer. 2001; 84:1591-1598. [PubMed: 11401310]

17. Antman K, Ayash L, Elias A, et al. A phase II study of high-dose cyclophosphamide, thiotepa, and carboplatin with autologous marrow support in women with measurable advanced breast cancer responding to standard-dose therapy. J Clin Oncol. 1992; 10:102-110. [PubMed: 1727912]

18. Elias AD, Ibrahim J, Richardson P, et al. The impact of induction duration and the number of highdose cycles on the long-term survival of women with metastatic breast cancer treated with highdose chemotherapy with stem cell rescue: an analysis of sequential phase I/II trials from the DanaFarber/Beth Israel STAMP program. Biol Blood Marrow Transplant. 2002; 8:198-205. [PubMed: 12017145]

19. Somlo G, Sniecinski I, Odom-Maryon T, et al. Effect of CD34 ${ }^{+}$selection and various schedules of stem cell reinfusion and granulocyte colony-stimulating factor priming on hematopoietic recovery after high-dose chemotherapy for breast cancer. Blood. 1997; 89:1521-1528. [PubMed: 9057632]

20. Somlo G, Chow W, Hamasaki V, et al. Tandem-cycle high-dose melphalan and cisplatin with peripheral blood progenitor cell support in patients with breast cancer and other malignancies. Biol Blood Marrow Transplant. 2001; 7:284-293. [PubMed: 11400951] 
21. Nieto Y, Shpall EJ. High-dose chemotherapy for high-risk primary and metastatic breast cancer: is another look warranted? Curr Opin Oncol. 2009; 21:150-157. [PubMed: 19532017]

22. Basser RL, O’Neill A, Martinelli G, et al. Multicycle dose-intensive chemotherapy for women with high-risk primary breast cancer: results of International Breast Cancer Study Group Trial 15-95. J Clin Oncol. 2006; 24:370-378. [PubMed: 16421418]

23. Coombes RC, Howell A, Emson M, et al. High-dose chemotherapy and autologous stem cell transplantation as adjuvant therapy for primary breast cancer patients with four or more lymph nodes involved: long-term results of an international randomised trial. Ann Oncol. 2005; 16:726734. [PubMed: 15817602]

24. Hanrahan EO, Broglio K, Frye D, et al. Randomized trial of high-dose chemotherapy and autologous hematopoietic stem cell support for high-risk primary breast carcinoma: follow-up at 12 years. Cancer. 2006; 106:2327-2336. [PubMed: 16639731]

25. Leonard RC, Lind M, Twelves C, et al. Conventional adjuvant chemotherapy versus single-cycle, autograft-supported, high-dose, late-intensification chemotherapy in high-risk breast cancer patients: a randomized trial. J Natl Cancer Inst. 2004; 96:1076-1083. [PubMed: 15265969]

26. Moore HC, Green SJ, Gralow JR, et al. Intensive dose-dense compared with high-dose adjuvant chemotherapy for high-risk operable breast cancer: Southwest Oncology Group/Intergroup Study 9623. J Clin Oncol. 2007; 25:1677-1682. [PubMed: 17404368]

27. Nitz UA, Mohrmann S, Fischer J, et al. Comparison of rapidly cycled tandem high-dose chemotherapy plus peripheral-blood stem-cell support versus dose-dense conventional chemotherapy for adjuvant treatment of high-risk breast cancer: results of a multicentre phase III trial. Lancet. 2005; 366:1935-1944. [PubMed: 16325695]

28. Peters WP, Rosner GL, Vredenburgh JJ, et al. Prospective, randomized comparison of high-dose chemotherapy with stem-cell support versus intermediate-dose chemotherapy after surgery and adjuvant chemotherapy in women with high-risk primary breast cancer: a report of CALGB 9082, SWOG 9114, and NCIC MA-13. J Clin Oncol. 2005; 23:2191-2200. [PubMed: 15767638]

29. Rodenhuis S, Bontenbal M, van Hoesel QG, et al. Efficacy of high-dose alkylating chemotherapy in HER2/neu-negative breast cancer. Ann Oncol. 2006; 17:588-596. [PubMed: 16446318]

30. Schrama JG, Faneyte IF, Schornagel JH, et al. Randomized trial of high-dose chemotherapy and hematopoietic progenitor-cell support in operable breast cancer with extensive lymph node involvement: final analysis with 7 years of follow-up. Ann Oncol. 2002; 13:689-698. [PubMed: 12075736]

31. Tokuda Y, Tajima T, Narabayashi M, et al. Phase III study to evaluate the use of high-dose chemotherapy as consolidation of treatment for high-risk postoperative breast cancer: Japan Clinical Oncology Group study, JCOG 9208. Cancer Sci. 2008; 99:145-151. [PubMed: 17970786]

32. Wilking N, Lidbrink E, Wiklund T, et al. Long-term follow-up of the SBG 9401 study comparing tailored FEC-based therapy versus marrow-supported high-dose therapy. Ann Oncol. 2007; 18:694-700. [PubMed: 17301072]

33. Zander AR, Schmoor C, Kroger N, et al. Randomized trial of high-dose adjuvant chemotherapy with autologous hematopoietic stem-cell support versus standard-dose chemotherapy in breast cancer patients with 10 or more positive lymph nodes: overall survival after 6 years of follow-up. Ann Oncol. 2008; 19:1082-1089. [PubMed: 18304964]

34. Tallman MS, Gray R, Robert NJ, et al. Conventional adjuvant chemotherapy with or without highdose chemotherapy and autologous stem-cell transplantation in high-risk breast cancer. $\mathrm{N}$ Engl $\mathbf{J}$ Med. 2003; 349:17-26. [PubMed: 12840088]

35. Gianni AM, Bonadonna G. Updated 12-year results of a randomized clinical trial comparing standard-dose to high-dose myeloablative chemotherapy in the adjuvant treatment of breast cancer with more than three positive nodes (LN+) [abstract]. J Clin Oncol. 2007; 25:549.

36. Roche HH, Pouillart P, Meyer N, et al. Adjuvant high-dose chemotherapy (HDC) improves early outcome for high-risk ( $\mathrm{N}>7$ ) breast cancer patients: the PEGASE 01 trial [abstract]. Proc Am Soc Clin Oncol. 2001; 20:102.

37. Farquhar C, Marjoribanks J, Lethaby A, et al. High-dose chemotherapy for poor prognosis breast cancers: systematic review and meta-analysis. Cancer Treat Rev. 2007; 33:325-337. [PubMed: 17382477] 
38. Berry DA, Ueno NT, Johnson MM, et al. High-dose chemotherapy with autologous stem-cell support as adjuvant therapy in breast cancer: overview of 15 randomized trials. J Clin Oncol. 2011; 29:3214-3223. [PubMed: 21768471]

39. Gluz O, Nitz UA, Harbeck N, et al. Triple-negative high-risk breast cancer derives particular benefit from dose intensification of adjuvant chemotherapy: results of the WSGAM-01 trial. Ann Oncol. 2008; 19:861-870. [PubMed: 18174609]

40. De Giorgi U, Rosti G, Frassinetti L, et al. High-dose chemotherapy for triple-negative breast cancer. Ann Oncol. 2007; 18:202-203. [PubMed: 16971660]

41. Biron P, Durand M, Roche H, et al. Pegase 03: a prospective randomized phase III trial of FEC with or without high-dose thiotepa, cyclophosphamide and autologous stem cell transplantation in first-line treatment of metastatic breast cancer. Bone Marrow Transplant. 2008; 41:555-562. [PubMed: 18037940]

42. Crump M, Gluck S, Tu D, et al. Randomized trial of high-dose chemotherapy with autologous peripheral-blood stem-cell support compared with standard-dose chemotherapy in women with metastatic breast cancer: NCIC MA.16. J Clin Oncol. 2008; 26:37-43. [PubMed: 18025439]

43. Lotz JP, Cure H, Janvier M, et al. High-dose chemotherapy with haematopoietic stem cell transplantation for metastatic breast cancer patients: final results of the French multicentric randomised CMA/PEGASE 04 protocol. Eur J Cancer. 2005; 41:71-80. [PubMed: 15617992]

44. Schmid P, Schippinger W, Nitsch T, et al. Up-front tandem high-dose chemotherapy compared with standard chemotherapy with doxorubicin and paclitaxel in metastatic breast cancer: results of a randomized trial. J Clin Oncol. 2005; 23:432-440. [PubMed: 15659490]

45. Stadtmauer EA, O’Neill A, Goldstein LJ, et al. Conventional-dose chemotherapy compared with high-dose chemotherapy plus autologous hematopoietic stem-cell transplantation for metastatic breast cancer. Philadelphia Bone Marrow Transplant Group. N Engl J Med. 2000; 342:1069-1076.

46. Vredenburgh JJ, Coniglio D, Broadwater G, et al. Consolidation with high-dose combination alkylating agents with bone marrow transplantation significantly improves disease-free survival in hormone-insensitive metastatic breast cancer in complete remission compared with intensive standard-dose chemotherapy alone. Biol Blood Marrow Transplant. 2006; 12:195-203. [PubMed: 16443517]

47. Vredenburgh JJ, Madan B, Coniglio D, et al. A randomized phase III comparative trial of immediate consolidation with high-dose chemotherapy and autologous peripheral blood progenitor cell support compared to observation with delayed consolidation in women with metastatic breast cancer and only bone metastases following intensive induction chemotherapy. Bone Marrow Transplant. 2006; 37:1009-1015. [PubMed: 16633363]

48. Berry DA, Ueno NT, Johnson MM, et al. High-dose chemotherapy with autologous hematopoietic stem-cell transplantation in metastatic breast cancer: overview of six randomized trials. J Clin Oncol. 2011; 29:3224-3231. [PubMed: 21768454] 


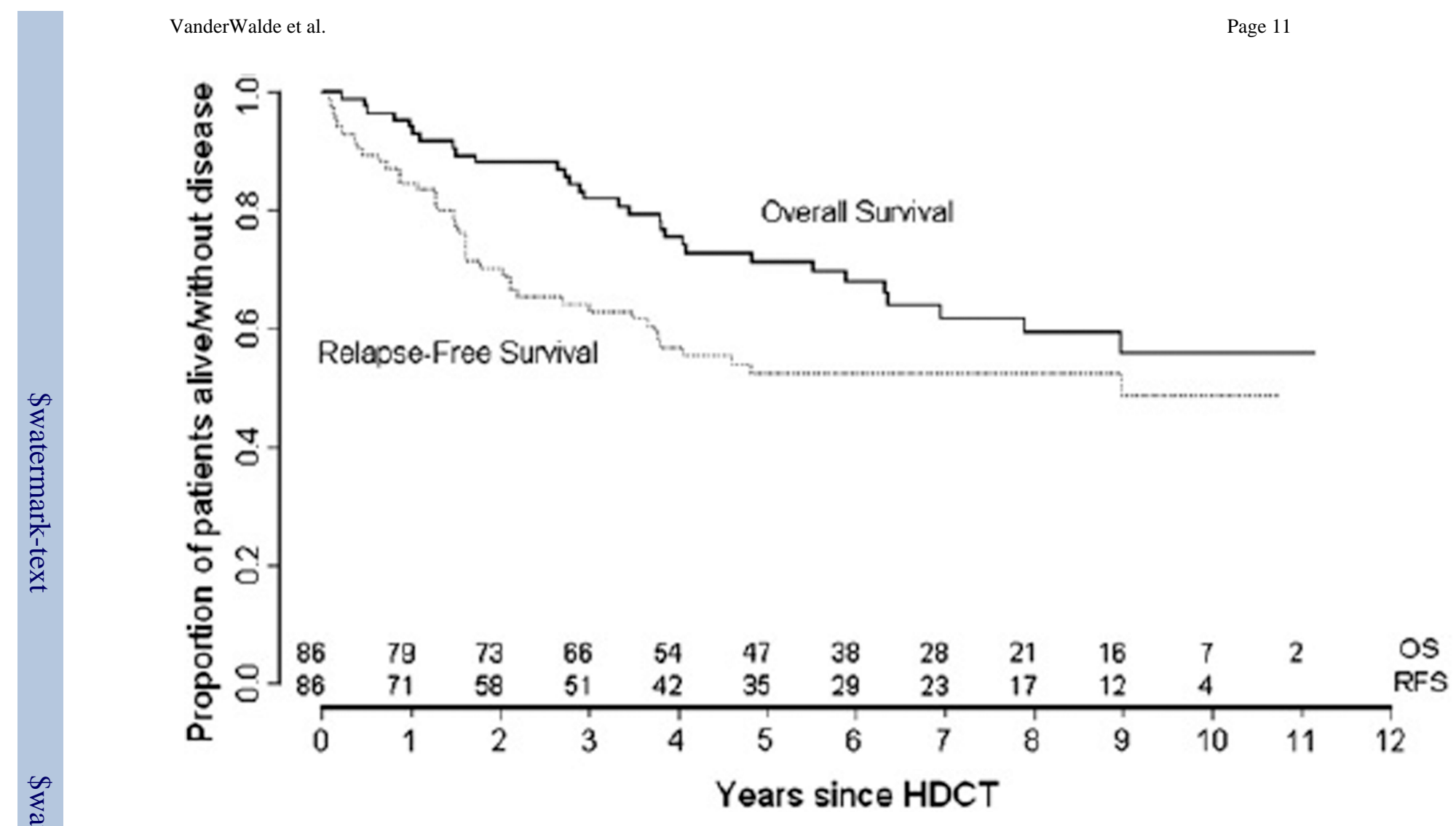

Figure 1.

RFS and OS for patients with stage III disease. 


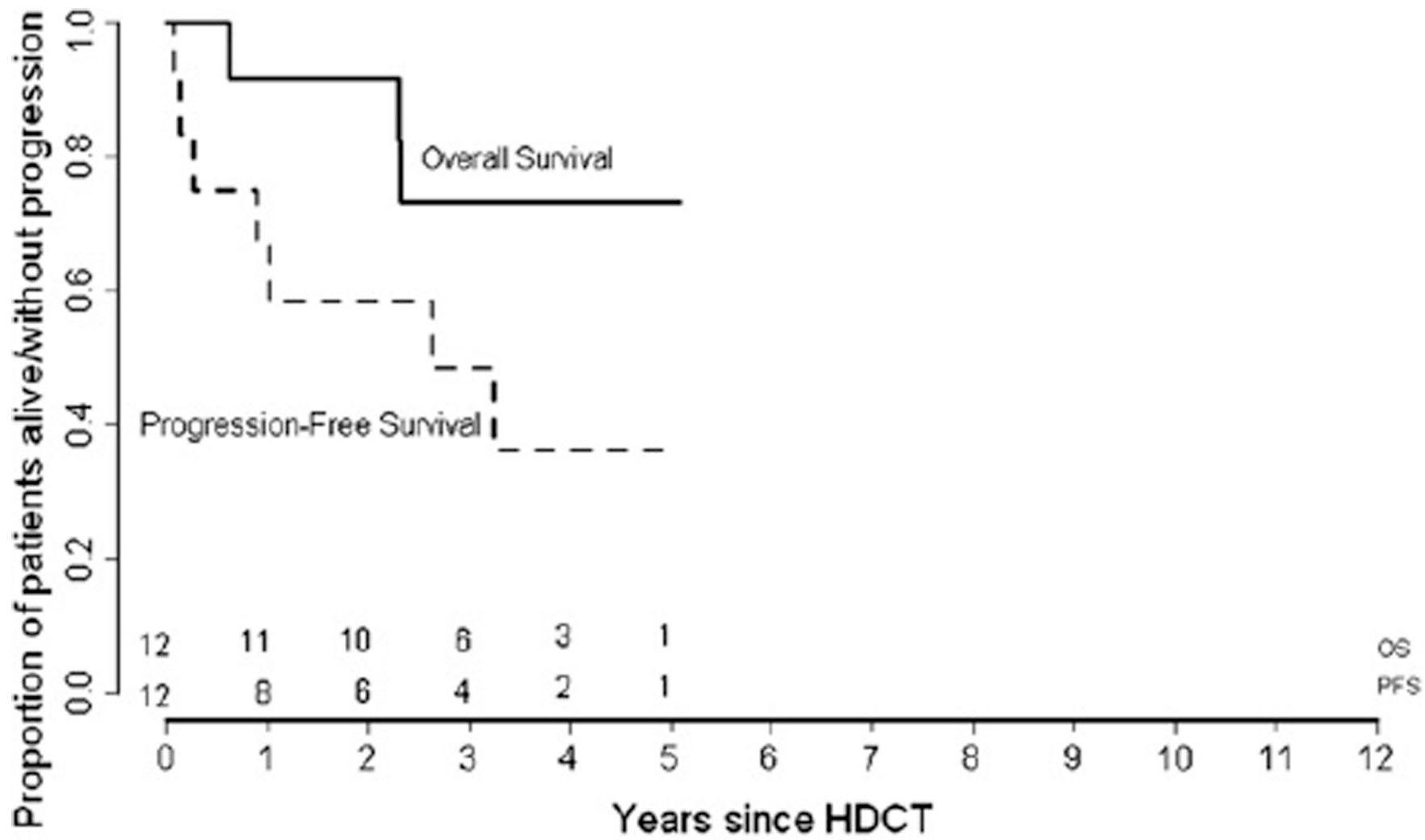

Figure 2.

PFS and OS for patients with oligometastatic (stage IV) disease. 
Table 1

Patient Characteristics

\begin{tabular}{|c|c|c|}
\hline Variable & Stage III $(n=86)$ & Stage IV $(n=86)$ \\
\hline \multicolumn{3}{|l|}{ Demographic characteristics, median (range) } \\
\hline Age, years & $50(28-66)$ & $44(32-57)$ \\
\hline Follow-up for living patients, months & $84(6-136)$ & $40(24-62)$ \\
\hline Time from diagnosis to protocol treatment, months & $8.1(4.6-39.2)$ & $21.3(7.3-106.4)$ \\
\hline \multicolumn{3}{|l|}{ Race, n (\%) } \\
\hline Asian & $3(3)$ & $2(17)$ \\
\hline Black & $3(3)$ & $1(8)$ \\
\hline Caucasian & $59(69)$ & $8(67)$ \\
\hline Hispanic & $21(24)$ & $1(8)$ \\
\hline \multicolumn{3}{|l|}{ Disease characteristics, $\mathrm{n}(\%)$} \\
\hline \multicolumn{3}{|l|}{ Stage } \\
\hline IIIA & $13(15)$ & 0 \\
\hline IIIB & $9(10)$ & 0 \\
\hline IIIC & $64(74)$ & 0 \\
\hline IV & 0 & $12(100)$ \\
\hline \multicolumn{3}{|l|}{$\mathrm{ER} / \mathrm{PR}$} \\
\hline Negative/negative & $22(26)$ & $1(8)$ \\
\hline Negative/positive & $1(1)$ & 0 \\
\hline Positive/negative & $12(14)$ & $1(8)$ \\
\hline Positive/positive & $51(59)$ & $10(83)$ \\
\hline \multicolumn{3}{|l|}{ HER2 } \\
\hline Negative & $56(65)$ & $9(75)$ \\
\hline Positive & $20(23)$ & $3(25)$ \\
\hline Missing & $10(12)$ & 0 \\
\hline \multicolumn{3}{|l|}{ Triple negative } \\
\hline No & $69(80)$ & $12(100)$ \\
\hline Yes & $15(17)$ & 0 \\
\hline Unknown & $2(2)$ & 0 \\
\hline \multicolumn{3}{|l|}{ Grade } \\
\hline 1 or 2 & $43(50)$ & $4(33)$ \\
\hline 3 & $43(50)$ & $7(58)$ \\
\hline Unknown & 0 & $1(8)$ \\
\hline \multicolumn{3}{|l|}{ Histology } \\
\hline Ductal & $67(78)$ & $12(100)$ \\
\hline Lobular & $19(22)$ & 0 \\
\hline \multicolumn{3}{|l|}{ Inflammatory } \\
\hline Yes & $16(19)$ & $1(8)$ \\
\hline No & $70(80)$ & $11(92)$ \\
\hline
\end{tabular}




\begin{tabular}{llc}
\hline Variable & Stage III $(\mathbf{n}=\mathbf{8 6})$ & Stage IV $(\mathbf{n}=\mathbf{8 6})$ \\
\hline Previous neoadjuvant chemotherapy & $32(37)$ & $2(17)$ \\
Radiation therapy received & $64(74)$ & $12(100)$ \\
Conditioning regimen & & \\
STAMP-V & $52(60)$ & 0 \\
ACT & $19(22)$ & 0 \\
Melphalan alone & $3(3)$ & $3(25)$ \\
Melphalan + STAMP-V & $12(14)$ & $9(75)$ \\
Outcomes, n (\%) & & \\
RFS/PFS & $63(52-72)$ & $49(19-73)$ \\
At 3 years & $53(41-63)$ & \\
At 5 years & & \\
OS & $82(72-89)$ & $73(38-91)$ \\
At 3 years & $71(60-80)$ & $-{ }^{*}$ \\
At 5 years & & \\
\hline
\end{tabular}

* Too few patients in stage IV had been progression-free or survived for 5 years. 
Table 2

Outcome by Univariate Analysis in Patients with Stage III Disease

\begin{tabular}{|c|c|c|c|}
\hline RFS & 5-Year DFS (95\% CI) & HR $(95 \%$ CI $)$ & $P$ value \\
\hline \multicolumn{4}{|l|}{ Stage } \\
\hline IIIA & $84 \%(49 \%-96 \%)$ & 1 & $.048^{*}$ \\
\hline IIIB & $63 \%(24 \%-87 \%)$ & $2.87(0.48-17.18)$ & \\
\hline IIIC & $45 \%(32 \%-57 \%)$ & $4.76(1.14-19.81)$ & \\
\hline \multicolumn{4}{|l|}{ ER/PR } \\
\hline Negative & $30 \%(12 \%-50 \%)$ & 1 & $.004^{*}$ \\
\hline Positive & $60 \%(47 \%-72 \%)$ & $0.4(0.21-0.76)$ & \\
\hline \multicolumn{4}{|l|}{ HER2 } \\
\hline Negative & $54 \%(39 \%-66 \%)$ & 1 & .87 \\
\hline Positive & $51 \%(26 \%-71 \%)$ & $1.20(0.58-2.50)$ & \\
\hline Missing & & $0.97(0.37-2.53)$ & \\
\hline \multicolumn{4}{|l|}{ Grade } \\
\hline 1 or 2 & $53 \%(37 \%-67 \%)$ & 1 & .97 \\
\hline 3 & $52 \%(36 \%-66 \%)$ & $1.01(0.54-1.88)$ & \\
\hline \multicolumn{4}{|l|}{ HDCT } \\
\hline $\mathrm{ACT}$ & $47 \%(24 \%-67 \%)$ & 1 & .55 \\
\hline STAMP-V & $55 \%(41 \%-68 \%)$ & $0.71(0.34-1.47)$ & \\
\hline Melphalan/STAMP-V ${ }^{\dagger}$ & $53 \%(23 \%-77 \%)$ & $1.03(0.38-2.81)$ & \\
\hline OS & $5-Y e a r$ OS $(95 \%$ CI $)$ & $\operatorname{HR}(95 \% \mathrm{CI})$ & $P$ value \\
\hline \multicolumn{4}{|l|}{ Stage } \\
\hline IIIA & $92 \%(54 \%-99 \%)$ & 1 & .11 \\
\hline IIIB & $89 \%(43 \%-98 \%)$ & $1.09(0.1-12.08)$ & \\
\hline IIIC & $65 \%(52 \%-76 \%)$ & $3.42(0.81-14.39)$ & \\
\hline \multicolumn{4}{|l|}{$\mathrm{ER} / \mathrm{PR}$} \\
\hline Negative & $38 \%(18 \%-58 \%)$ & 1 & $<.001 *$ \\
\hline Positive & $83 \%(70 \%-90 \%)$ & $0.26(0.13-0.54)$ & \\
\hline \multicolumn{4}{|l|}{ HER2 } \\
\hline Negative & $73 \%(59 \%-83 \%)$ & 1 & .61 \\
\hline Positive & $67 \%(41 \%-84 \%)$ & $1.49(0.66-3.34)$ & \\
\hline Missing & $70 \%(33 \%-89 \%)$ & $1.03(0.34-3.09)$ & \\
\hline \multicolumn{4}{|l|}{ Grade } \\
\hline 1 or 2 & $77 \%(60 \%-87 \%)$ & 1 & .18 \\
\hline 3 & $66 \%(49 \%-78 \%)$ & $1.65(0.79-3.43)$ & \\
\hline \multicolumn{4}{|l|}{ HDCT } \\
\hline $\mathrm{ACT}$ & $63 \%(38 \%-80 \%)$ & 1 & .61 \\
\hline STAMP-V & $75 \%(60 \%-84 \%)$ & $0.67(0.31-1.48)$ & \\
\hline Melphalan/STAMP-V ${ }^{\dagger}$ & $75 \%(41 \%-92 \%)$ & $0.86(0.23-3.24)$ & \\
\hline
\end{tabular}

Biol Blood Marrow Transplant. Author manuscript; available in PMC 2013 August 01. 
Difference attained statistical significance.

Patients intended to be treated with melphalan + STAMP-V. 


\section{Table 3}

Multivariate Cox Regression Models for Patients with Locally Advanced Disease

\begin{tabular}{llcc}
\hline Outcome & Variable & HR $(\mathbf{9 5 \%}$ CI) & $\boldsymbol{P}$ value \\
\hline RFS & Stage (IIIC versus IIIA/B) & $3.04(1.19-7.78)$ & .020 \\
& ER/PR (+ versus -) & $0.38(0.20-0.73)$ & .004 \\
\multirow{2}{*}{ OS } & Stage (IIIC versus IIIA/B) & $3.30(1.00-10.9)$ & .05 \\
& ER/PR (+ versus -) & $0.26(0.13-0.54)$ & $<.001$ \\
\hline
\end{tabular}


Table 4

Grade 3 and Higher Nonhematologic Toxicities Associated with HDCT

\begin{tabular}{|c|c|c|c|}
\hline Toxicity & $\begin{array}{l}\text { ACT, } \\
\mathbf{n}(\%)\end{array}$ & $\begin{array}{l}\text { STAMP-V, } \\
\text { n(\%) }\end{array}$ & $\begin{array}{l}\text { Melphalan + } \\
\text { STAMP-V, n (\%) }\end{array}$ \\
\hline Altered mental status & $3(16)^{\dagger}$ & $1(2)$ & $0(0)$ \\
\hline Anorexia/weight loss & $6(32)$ & $11(21)$ & $11(41)$ \\
\hline Diarrhea & $0(0)$ & $0(0)$ & $5(19)$ \\
\hline Dyspnea (shortness of breath) & $0(0)$ & $2(4)$ & $0(0)$ \\
\hline Edema/weight gain & $0(0)$ & $2(4)$ & $0(0)$ \\
\hline Electrolyte abnormalities & $16(84)$ & $26(50)$ & $12(44)$ \\
\hline Esophagitis & $15(79)$ & $2(4)$ & $1(4)$ \\
\hline Fatigue (asthenia, lethargy, malaise) & $1(5)$ & $7(13)$ & $10(37)$ \\
\hline Febrile neutropenia & $16(84)$ & $25(48)$ & $14(52)$ \\
\hline Hemorrhage & $5(26)$ & $7(13)$ & $2(7)$ \\
\hline Hypertension & $2(11)$ & $2(4)$ & $0(0)$ \\
\hline Hypotension & $0(0)$ & $3(6)$ & $1(4)$ \\
\hline Hypoxia & $1(5)$ & $4(8)$ & $1(4)$ \\
\hline Infection/sepsis & $3(16)$ & $10(19)$ & $8(30)^{*}$ \\
\hline Liver dysfunction/failure & $2(11)$ & $0(0)$ & $1(4)$ \\
\hline Mucositis & $16(84)$ & $3(6)$ & $9(33)$ \\
\hline Myalgia & $1(5)$ & $2(4)$ & $3(11)$ \\
\hline Nausea/vomiting & $4(21)$ & $13(25)$ & $10(37)$ \\
\hline Neuropathy & $2(11)$ & $0(0)$ & $0(0)$ \\
\hline Pain, other & $1(5)$ & $4(8)$ & $4(15)$ \\
\hline Pain, bone/joint & $2(11)$ & $0(0)$ & $3(11)$ \\
\hline Rash & $0(0)$ & $2(4)$ & $0(0)$ \\
\hline Renal failure & $0(0)$ & $0(0)$ & $1(4)$ \\
\hline Second malignancy & $0(0)$ & $0(0)$ & $1(4)^{\xi}$ \\
\hline Syndrome of inappropriate secretion of antidiuretic hormone & $0(0)$ & $2(4)$ & $0(0)$ \\
\hline
\end{tabular}

* Patients treated with melphalan alone were combined with those treated with melphalan + STAMP-V.

Due to narcotic use for treatment of mucositis.

tone patient in the tandem group died of transplantation-related sepsis.

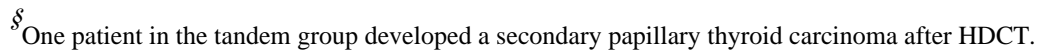

